\title{
The Dolbeault operator on Hermitian spin surfaces
}

\author{
B.Alexandrov G. Grantcharov S.Ivanov * \\ Department of Mathematics \\ University OF SOFIA \\ "ST. KL. OHRIDSKI"
}

\begin{abstract}
We consider the Dolbeault operator $\sqrt{2}\left(\bar{\partial}+\bar{\partial}^{*}\right)$ of $K^{\frac{1}{2}}$ - the square root of the canonical line bundle which determines the spin structure of a compact Hermitian spin surface $(M, g, J)$. We prove that all cohomology groups $H^{i}\left(M, \mathcal{O}\left(K^{\frac{1}{2}}\right)\right)$ vanish if the scalar curvature of $g$ is non-negative and non-identically zero. Moreover, we estimate the first eigenvalue of the Dolbeault operator when the conformal scalar curvature $k$ is non-negative and when $k$ is positive. In the first case we give a complete list of limiting manifolds and in the second one we give non-Kähler examples of limiting manifolds.
\end{abstract}

Running title: The Dolbeault operator on Hermitian spin surfaces

Keywords. Hermitian surface, Dirac operator, Dolbeault operator, twistor spinors

MS classification: $53 \mathrm{C} 15 ; 53 \mathrm{C} 25 ; 53 \mathrm{~B} 35$

${ }^{*}$ The authors are supported by Contract MM 809/1998 with the Ministry of Science and Education of Bulgaria and by Contract 238/1998 with the University of Sofia "St. Kl. Ohridski". 


\section{Introduction}

The well-known vanishing theorem of Lichnerowicz says that there are no harmonic spinors on compact spin manifolds of non-negative non-identically zero scalar curvature, i.e. the kernel of the Dirac operator vanishes. When the scalar curvature is identically zero the harmonic spinors are actually parallel. Complete classification of the complete simply connected irreducible spin manifolds admitting parallel spinor is given by Hitchin [14] and Wang [21]. When the scalar curvature is strictly positive one may try to find an estimate for the first eigenvalue of the Dirac operator. This is done by Friedrich [7]. The estimate is expressed in terms of the scalar curvature and the limiting manifolds are characterized by the existence of a real Killing spinor.

It is well-known (see [14]) that in the Kähler case the Dirac operator coincides with the Dolbeault operator $\square=\sqrt{2}\left(\bar{\partial}+\bar{\partial}^{*}\right)$ on $K^{\frac{1}{2}}$ - the square root of the canonical line bundle $K$ which determines the spin structure. Applying Hodge theory to the corresponding Dolbeault complex Hitchin 14] has shown that on a compact Kähler spin manifold the space of harmonic spinors can be identified with the holomorphic cohomology $H^{*}\left(M, \mathcal{O}\left(K^{\frac{1}{2}}\right)\right)$. So, if a compact Kähler spin manifold admits a Riemannian metric of strictly positive scalar curvature then all cohomology groups $H^{*}\left(M, \mathcal{O}\left(K^{\frac{1}{2}}\right)\right)$ vanish by Lichnerowicz theorem.

The purpose of this note is to treat problems similar to the above mentioned in the case of the Dolbeault operator on compact Hermitian spin surfaces.

Our first observation is the following.

Theorem 1.1 Let $(M, J)$ be a compact complex spin surface admitting a Hermitian metric of non-negative non-identically zero scalar curvature. Then $H^{i}\left(M, \mathcal{O}\left(K^{\frac{1}{2}}\right)\right)=0, i=0,1,2$.

Proof: By arguments similar to those in Proposition I.18 in [11] (see also Lemma 3.3 in [1]) the existence of a Hermitian metric of non-negative non-identically zero scalar curvature implies that all the plurigenera of $(M, J)$ vanish. Hence, $H^{0}\left(M, \mathcal{O}\left(K^{\frac{1}{2}}\right)\right)=0$. By Serre duality $H^{2}\left(M, \mathcal{O}\left(K^{\frac{1}{2}}\right)\right)=0$. By Lichnerowicz vanishing theorem the index of the Riemannian Dirac operator $D$ vanishes. But since both $D$ and the Dolbeault operator $\square$ are generalized Dirac operators on the same Clifford module, they have the same index. Thus,

$$
0=\operatorname{ind}(\square)=\operatorname{dim} H^{0}\left(M, \mathcal{O}\left(K^{\frac{1}{2}}\right)\right)-\operatorname{dim} H^{1}\left(M, \mathcal{O}\left(K^{\frac{1}{2}}\right)\right)+\operatorname{dim} H^{2}\left(M, \mathcal{O}\left(K^{\frac{1}{2}}\right)\right)
$$

and therefore $H^{1}\left(M, \mathcal{O}\left(K^{\frac{1}{2}}\right)\right)=0$.

In view of this result the following questions are natural:

Is there an estimate for the first eigenvalue of the Dolbeault operator on Hermitian spin surfaces of positive scalar curvature? If the answer is 'Yes', describe the limiting manifolds, i.e. the manifolds for which the estimate is attained.

For Kähler manifolds the above questions are treated in terms of the Dirac operator. However, according to the result of Hijazi [13] the estimate of Friedrich [7] is not sharp on Kähler manifold since it admits a parallel form. A better estimate for the first eigenvalue of the Dirac operator on compact Kähler spin manifold with strictly positive scalar curvature is found by Kirchberg [15, 16] and the limiting manifolds are characterized by the existense of Kählerian twistor spinors. In the 4-dimensional case (in which we are interested in this paper) the classification of limiting Kähler manifolds is given by Friedrich [8] using detailed study of the Kählerian twistor equations. So, the complete answer to both of the questions for compact Kähler spin surface is known. 
In the present paper we give complete answer to the questions under the stronger assumption of non-negative conformal scalar curvature. We recall that the conformal scalar curvature of a Hermitian surface is the scalar curvature of the corresponding Weyl connection. In the case of strictly positive conformal scalar curvature we answer to the first question completely and partially to the second.

Our considerations are based on Bochner type calculations using the set of canonical Hermitian connections $\nabla^{t}, t \in R$, described by Gauduchon [12]. Among these connections an important role plays the Bismut connection. This is the unique Hermitian connection with skew-symmetric torsion (cf. [12]) and is used by Bismut [5] to express the Weitzenböck formula for the Dolbeault operator. To treat limiting manifolds we consider twistor equations with respect to the canonical Hermitian connections.

More precisely, we prove

Theorem 1.2 Let $(M, g, J)$ be a compact Hermitian spin surface of non-negative conformal scalar curvature. Then the first eigenvalue $\lambda$ of the Dolbeault operator satisfies the inequality

$$
\lambda^{2} \geq \frac{1}{6} \inf _{M} s
$$

where $s$ is the scalar curvature of $g$. Further, the following conditions are equivalent:

(i) There is an equality in (1.1).

(ii) There exists a parallel spinor in $\Sigma_{+} M$ with respect to the Bismut connection, the conformal scalar curvature is identically zero and the scalar curvature is constant.

(iii) $(M, g, J)$ is a K3-surface or a flat torus with their hyper-Kähler metric or a coordinate quaternionic Hopf surface (see [6]) with a metric of constant scalar curvature in the conformal class of the standard locally conformally flat metric.

Theorem 1.3 Let $(M, g, J)$ be a compact Hermitian spin surface of positive conformal scalar curvature $k$. Then the first eigenvalue $\lambda$ of the Dolbeault operator satisfies the inequality

$$
\lambda^{2} \geq \frac{1}{2} \inf _{M} k
$$

The equality in (1.9) is attained iff $k$ is constant and there exists a Hermitian twistor spinor with respect to the Hermitian connection $\nabla^{-3}$. In this case $(M, g, J)$ is locally conformally Kähler.

Note that on Kähler surfaces the estimate (1.2) coincides with that of 16.

In the last section we give examples of non-Kähler Hermitian surfaces for which the limiting case of the inequality (1.2) is attained.

\section{Preliminaries}

Let $(M, g, J)$ be a Hermitian surface with complex structure $J$ and compatible metric $g$. Denote by $\Omega$ the Kähler form, defined by $\Omega(X, Y)=g(X, J Y)$. The volume form of $\mathrm{g}$ is $\omega=\frac{1}{2} \Omega \wedge \Omega$. It is well-known that $d \Omega=\theta \wedge \Omega$, where $\theta=\delta \Omega \circ J$ is the Lee form of $(M, g, J)$. Recall that $(M, g, J)$ is Kähler iff $\theta=0$; locally conformally Kähler iff $d \theta=0$; globally conformally Kähler iff $\theta=d f$ for a smooth function $f$ on $M$ (in this case $e^{-f} g$ is a Kähler metric). Let $\nabla$ be the 
Levi-Civita connection of $g$ and $R$ and $s$-its curvature tensor and scalar curvature respectively (for the curvature tensor we adopt the following definition: $R(X, Y, Z, W)=g\left(\left[\nabla_{X}, \nabla_{Y}\right] Z-\right.$ $\left.\left.\nabla_{[X, Y]} Z, W\right)\right)$. Recall that the ${ }^{*}$-Ricci tensor $\rho^{*}$ and the ${ }^{*}$-scalar curvature $s^{*}$ of $M$ are defined by

$$
\begin{gathered}
\rho^{*}(X, Y)=\sum_{i=1}^{4} R\left(e_{i}, X, J Y, J e_{i}\right)=-\frac{1}{2} \sum_{i=1}^{4} R\left(X, J Y, e_{i}, J e_{i}\right) \\
s^{*}=\sum_{i=1}^{4} \rho^{*}\left(e_{i}, e_{i}\right),
\end{gathered}
$$

where here and in the following $\left\{e_{i}\right\}$ is a local orthonormal frame of the tangent bundle $T M$ and $\left\{e^{i}\right\}$ is its dual frame.

We also have (cf. 20])

$$
s-s^{*}=2 \delta \theta+|\theta|^{2}
$$

Note that on a Kähler manifold the Ricci and ${ }^{*}$-Ricci tensors coincide; in particular $s=s^{*}$.

Now consider the Weyl connection determined by the Hermitian structure on $M$, i.e. the unique torsion-free connection $\nabla^{W}$ such that $\nabla^{W} g=\theta \otimes g$. The conformal scalar curvature $k$ is defined to be the scalar curvature of $\nabla^{W}$. Equivalently, $k$ is the ${ }^{*}$-scalar curvature of the self-dual Weyl tensor $W_{+}$, multiplied by $\frac{3}{2}$. The Weyl connection is invariant under conformal changes of the metric since if $\widetilde{g}=e^{f} g$ then $\widetilde{\theta}=\theta+d f$. Hence,

$$
\tilde{k}=e^{-f} k
$$

We also have

$$
k=\frac{3 s^{*}-s}{2}
$$

Recall the definition of the set of canonical Hermitian connections [12]: For a real number $t$ the connection $\nabla^{t}$ is defined by

$$
g\left(\nabla_{X}^{t} Y, Z\right)=g\left(\nabla_{X} Y, Z\right)-\frac{1}{2} g\left(\left(\nabla_{J X} J\right) Y, Z\right)+\frac{t}{4}(d \Omega(J X, J Y, J Z)+d \Omega(J X, Y, Z))
$$

or equivalently

$$
\begin{aligned}
\nabla_{X}^{t} Y= & \nabla_{X} Y-\frac{t+1}{4} \theta(Y) X+\frac{t-1}{4} \theta(J Y) J X-\frac{t}{2} \theta(J X) J Y \\
& +\frac{t+1}{4} g(X, Y) \theta^{\#}-\frac{t-1}{4} g(X, J Y) J \theta^{\#},
\end{aligned}
$$

where $\theta^{\#}$ is the vector field dual to $\theta$.

The canonical Hermitian connections form an affine line (degenerating to a point in the Kähler case) determined by $\nabla^{0}$ - the projection of the Levi-Civita connection into the affine space of all Hermitian connections, and $\nabla^{1}$, which coincides with the Chern connection. In the sequel important role will be played also by the connections $\nabla^{-1}$ (considered by Bismut [5]) and $\nabla^{-3}$.

From now on we assume that $(M, g, J)$ is spin manifold. Denote by $\Sigma M$ its spinor bundle and let $\mu: T^{*} M \otimes \Sigma M \longrightarrow \Sigma M$ be the Clifford multiplication. Identifying $T^{*} M$ and $T M$ via the metric we shall also consider $\mu$ as a map from $T M \otimes \Sigma M$ into $\Sigma M$. We shall often write the Clifford multiplication by juxtaposition, i.e.

$$
\mu(\alpha \otimes \psi)=\alpha \psi, \quad \mu(X \otimes \psi)=X \psi, \quad \alpha \in T^{*} M, \quad X \in T M, \quad \psi \in \Sigma M .
$$


Since $(M, g, J)$ is a Hermitian manifold the choice of a spin structure on it is equivalent to the choice of a square-root $K^{\frac{1}{2}}$ of the canonical line bundle $K$ [14]. Thus for the corresponding spinor bundle we have

$$
\Sigma M=\Lambda^{0, \bullet} M \otimes K^{\frac{1}{2}},
$$

where $\Lambda^{0, \bullet} M=\Lambda^{0,0} M \oplus \Lambda^{0,1} M \oplus \Lambda^{0,2} M$. In particular, the spinor bundle $\Sigma M$ splits as follows:

$$
\Sigma M=\Sigma_{0} M \oplus \Sigma_{1} M \oplus \Sigma_{2} M
$$

where $\Sigma_{r} M=\Lambda^{0, r} M \otimes K^{\frac{1}{2}}$ is the eigensubbundle with respect to the eigenvalue $(2-2 r) i$ of the Clifford action of the Kähler form $\Omega$ on $\Sigma M$ (cf. [15]).

The half-spinor bundles $\Sigma_{ \pm} M$ are the eigensubbundles of the volume form $\omega$ with respect to its eigevalues $\mp 1$ and we have

$$
\Sigma_{+} M=\Sigma_{0} M \oplus \Sigma_{2} M, \quad \Sigma_{-} M=\Sigma_{1} M .
$$

Let $p_{r}: \Sigma M \longrightarrow \Sigma_{r} M, r=0,1,2$, be the projections with respect to the splitting (2.8). For convenience we denote $\Sigma_{-1} M=\Sigma_{3} M=0$ and $p_{-1}=p_{3}=0$. Recall [15] that for $X \in T M$

$$
X \Omega-\Omega X=2 J X
$$

as endomorphisms of $\Sigma M$ and for $\psi \in \Sigma_{r} M$

$$
\begin{gathered}
X \psi=p_{r-1} X \psi+p_{r+1} X \psi, \quad J X \psi=-i p_{r-1} X \psi+i p_{r+1} X \psi \\
p(X) \psi=p_{r+1} X \psi, \quad \bar{p}(X) \psi=p_{r-1} X \psi,
\end{gathered}
$$

where $p(X)=\frac{1}{2}(X-i J X), \bar{p}(X)=\frac{1}{2}(X+i J X)$.

Any metric connection in the tangent bundle gives rise to metric connection in the spinor bundle and it is easy consequence of (2.6) that

$$
\nabla_{X}^{t} \psi=\nabla_{X} \psi+\frac{1}{8}[(t+1) X \theta+(t-1) J X J \theta+2 t \theta(X)+2 t \theta(J X) \Omega] \psi
$$

for $X \in \Gamma(T M), \quad \psi \in \Gamma(\Sigma M)$. In particular, we have

$$
\nabla_{X}^{t} \psi_{0}=\nabla_{X} \psi_{0}+\frac{1}{4} X \theta \psi_{0}+\frac{1}{4} \theta(X) \psi_{0}+\frac{t+1}{4} i \theta(J X) \psi_{0}
$$

for $X \in \Gamma(T M), \quad \psi_{0} \in \Gamma\left(\Sigma_{0} M\right)$. This follows from (2.12) and

$$
p_{0} X \alpha \psi_{0}=-2 \alpha(\bar{p}(X)) \psi_{0} \quad X \in \Gamma(T M), \quad \alpha \in \Gamma\left(T^{*} M\right), \quad \psi_{0} \in \Gamma\left(\Sigma_{0} M\right) .
$$

The Kähler form $\Omega$ is parallel with respect to any Hermitian connection, so the Hermitian connections preserve the splitting (2.8). Recall also [3] that there is an antilinear bundle map $j: \Sigma M \longrightarrow \Sigma M$ which commutes with the Clifford multiplication by real vectors, $j^{2}=-1, j$ is parallel with respect to any metric connection on $M$ and preserves the Hermitian inner product on $\Sigma M$. In particular, $j$ provides an antilinear isomorphism between $\Sigma_{0} M$ and $\Sigma_{2} M$.

The Dirac operator of the Levi-Civita connection $D: \Gamma(\Sigma M) \longrightarrow \Gamma(\Sigma M)$ is defined by $D=\mu \circ \nabla$ or equivalently by $D \psi=\sum_{i=1}^{4} e^{i} \nabla_{e_{i}} \psi$. The Dirac operators $D^{t}$ of the connections $\nabla^{t}$ are defined in similar way by replacing $\nabla$ with $\nabla^{t}$. 
The identification $(2.7)$ shows that the Dolbeault operator $\square=\sqrt{2}\left(\bar{\partial}+\bar{\partial}^{*}\right)$ of $K^{\frac{1}{2}}$ also acts on sections of the spinor bundle. As shown in [12]

$$
\begin{gathered}
D^{t}=D-\frac{3 t}{4} \theta-\frac{2 t-1}{4} J \theta \circ \Omega \\
\square=D+\frac{1}{4} \theta+\frac{1}{4} J \theta \circ \Omega
\end{gathered}
$$

Let $\pi_{r}:\left.T^{*} M \otimes \Sigma_{r} M \longrightarrow \operatorname{Ker} \mu\right|_{T^{*} M \otimes \Sigma_{r} M}, r=0,1,2$, be the orthogonal projections. It is easy to see that

$$
\begin{aligned}
& \pi_{r}(\alpha \otimes \psi) \\
& =\alpha \otimes \psi+\frac{1}{2(r+1)} \sum_{i=1}^{4} e^{i} \otimes \bar{p}\left(e^{i}\right) p(\alpha) \psi+\frac{1}{4-2(r-1)} \sum_{i=1}^{4} e^{i} \otimes p\left(e^{i}\right) \bar{p}(\alpha) \psi,
\end{aligned}
$$

where $p(\alpha)=\frac{1}{2}(\alpha-i J \alpha), \bar{p}(\alpha)=\frac{1}{2}(\alpha+i J \alpha)$ and $J \alpha$ is the dual form of $J \alpha^{\#}, J \alpha=-\alpha \circ J$.

The twistor operators of the Hermitian connection $\nabla^{t}$ are the differential operators $P_{r}^{t}: \Gamma\left(\Sigma_{r} M\right) \longrightarrow \Gamma\left(T^{*} M \otimes \Sigma_{r} M\right)$ defined by $P_{r}^{t}=\pi_{r} \circ \nabla^{t}$. By analogy with the Kähler twistor spinors of [16] we shall call the spinors in the kernel of $P_{r}^{t}$ Hermitian twistor spinors with respect to $\nabla^{t}$. We are particularly interested in $P_{0}^{t}$. It follows from (2.17) that

$$
P_{0}^{t} \psi_{0}=\nabla^{t} \psi_{0}+\frac{1}{2} \sum_{i=1}^{4} e^{i} \otimes p_{0} e_{i} D^{t} \psi_{0}, \quad \psi_{0} \in \Gamma\left(\Sigma_{0} M\right)
$$

and also

$$
P_{0}^{t} \psi_{0}=\sum_{i=1}^{4} e^{i} \otimes \nabla_{p\left(e_{i}\right)}^{t} \psi_{0}=\left(\nabla^{t}\right)^{1,0} \psi_{0}, \quad \psi_{0} \in \Gamma\left(\Sigma_{0} M\right) .
$$

In the 4-dimensional case the Weitzenböck formula for the Dolbeault operator ( [5], Theorem 2.3) reads as follows:

$$
\square^{2}=\Delta^{-1}+\frac{s}{4}+\frac{1}{4} \delta \theta \cdot \omega-\frac{|\theta|^{2}}{8},
$$

where $\Delta^{t}=\left(\nabla^{t}\right)^{*} \nabla^{t}$ is the spinor Laplacian of the connection $\nabla^{t}$.

In the following we shall denote by $\langle.,$.$\rangle and |$.$| pointwise inner products and norms and$ by $(.,$.$) and \|\|-$. the global ones respectively.

\section{Proof of Theorem 1.2 and Theorem 1.3}

Throughout this section $(M, g, J)$ is a compact Hermitian spin surface.

Lemma 3.1 Let $\lambda \neq 0$ be an eigenvalue of the Dolbeault operator $\square$. Then there exists an eigenspinor $\psi_{0} \in \Gamma\left(\Sigma_{0} M\right)$ for the eigenvalue $\lambda^{2}$ of $\square^{2}$.

Proof: It is clear that $\square\left(\Gamma\left(\Sigma_{r} M\right)\right) \subset \Gamma\left(\Sigma_{r-1} M\right) \oplus \Gamma\left(\Sigma_{r+1} M\right)$, and $\square^{2}\left(\Gamma\left(\Sigma_{r} M\right)\right) \subset \Gamma\left(\Sigma_{r} M\right)$, $r=0,1,2$. Hence, if $\psi \neq 0$ is an eigenspinor of $\square$ for $\lambda$ and $\psi=\psi_{0}+\psi_{1}+\psi_{2}$ with respect to the decomposition (2.8) then

$$
\psi=\left(\psi_{0}+\frac{1}{\lambda} \square \psi_{0}\right)+\left(\psi_{2}+\frac{1}{\lambda} \square \psi_{2}\right)
$$


(i.e. $\psi_{1}=\frac{1}{\lambda} \square \psi_{0}+\frac{1}{\lambda} \square \psi_{2}$ ) and both $\psi_{0}+\frac{1}{\lambda} \square \psi_{0}$ and $\psi_{2}+\frac{1}{\lambda} \square \psi_{2}$ are eigenspinors for $\square$ with respect to $\lambda$. Moreover, $\psi_{0}, \square \psi_{0}, \psi_{2}, \square \psi_{2}$ are eigenspinors for $\square^{2}$ with respect to $\lambda^{2}$. It follows by (2.16) that $\square \circ j=j \circ \square$ and therefore $j \psi_{2} \in \Gamma\left(\Sigma_{0} M\right)$ is also an eigenspinor for $\square^{2}$ with respect to $\lambda^{2}$. Since by (3.21) $\psi_{0} \neq 0$ or $\psi_{2} \neq 0$, Lemma 3.1 is proved.

Proof of Theorem 1.9: When $\inf _{M} s \leq 0$ the inequality (1.1) is trivially satisfied. So we can assume that $\inf _{M} s>0$. Hence it follows from Theorem 1.1 that $\lambda \neq 0$ and by Lemma 3.1 we have an eigenspinor $\psi_{0} \in \Gamma\left(\Sigma_{0} M\right)$ of $\square^{2}$ with respect to $\lambda^{2}$. Thus $(2.20)$ yields

$$
\lambda^{2}\left\|\psi_{0}\right\|^{2}=\left(\square^{2} \psi_{0}, \psi_{0}\right)=\left(\Delta^{-1} \psi_{0}, \psi_{0}\right)+\frac{1}{4}\left(\left(s-\delta \theta-\frac{|\theta|^{2}}{2}\right) \psi_{0}, \psi_{0}\right) .
$$

By (2.3) $s-\delta \theta-\frac{|\theta|^{2}}{2}=\frac{s+s^{*}}{2}$ and since $k \geq 0$, i.e. $s^{*} \geq \frac{s}{3}$, we obtain that $\lambda^{2} \geq \frac{1}{6} \inf _{M} s$.

Now we proceed with the proof of the second part of the theorem. Note that the Bismut connection restricted to sections of $\Sigma_{+} M$ coincides with the connection considered in [18] and called there "the Weyl connection". Hence, as proved in [18], a parallel spinor in $\Sigma_{+} M$ with respect to the Bismut connection gives rise to a hyper-Hermitian structure on $M$ and thus $M$ is conformally equivalent to one of the manifolds in (iii) (cf. also [6]). In particular, it follows that $M$ is anti-self-dual, which is equivalent to $k=0$ and $d \theta=0$ (cf. for example [1]). Conversely, any manifold conformally equivalent to those listed in (iii) admits a parallel spinor in $\Sigma_{+} M$ (and hence in $\Sigma_{0} M$ ) with respect to the Bismut connection in the spin structure given by the trivial square-root of the canonical line bundle. This proves the equivalence of (ii) and (iii) since the hyper-Kähler metrics on a $K 3$-surface or a torus are the only metrics of constant scalar curvature in their conformal classes.

Now we prove the equivalence of (i) and (ii).

When $s>0$ the calculations in the first part of the proof show that there is an equality in (1.1) iff $s=$ const, $k=0$ and $\nabla^{-1} \psi_{0}=0$. But as shown above, $\nabla^{-1} \psi_{0}=0$ implies $k=0$ and thus (i) and (ii) are equivalent when $s>0$.

When $\inf _{M} s=0$ Theorem 1.1 shows that the equality in (1.1) is possible only if $s=0$. Hence, by $k \geq 0$ we have $s^{*} \geq 0$ and integrating (2.3) we obtain that $\theta=0$, i.e. $(M, g, J)$ is Kähler. Therefore (2.20) (which in the Kähler case coincides with the usual Lichnerowicz formula and the Bismut connection coincides with Levi-Civita connection) yields that $\square^{2} \psi=0$ iff $\nabla^{-1} \psi=0$. Hence it remains to show that if there exists a parallel spinor, then there exists a parallel spinor in $\Sigma_{+} M$. But $(M, g, J)$ is anti-self-dual (since $k=s=0$ and $d \theta=0$ ) and thus its signature $\sigma(M) \leq 0$. Since $\sigma(M)=-\operatorname{sind}(D)=-8 \operatorname{ind}(\square)$ it follows that $\operatorname{ind}(\square) \geq 0$, i.e. $\operatorname{dimKer}\left(\left.\square\right|_{\Sigma_{+} M}\right) \geq \operatorname{dimKer}\left(\left.\square\right|_{\Sigma_{-} M}\right)$.

Thus the equivalence of (i) and (ii) is proved.

We start the proof of Theorem 1.3 by two Lemmas.

Lemma 3.2 Let $\psi_{0} \in \Gamma\left(\Sigma_{0} M\right)$. Then

$$
\begin{aligned}
\left\|P_{0}^{t} \psi_{0}\right\|^{2}= & \frac{1}{2}\left(\square^{2} \psi_{0}, \psi_{0}\right)-\frac{1}{4}\left(s \psi_{0}, \psi_{0}\right)-\frac{t}{4}\left(\delta \theta \psi_{0}, \psi_{0}\right) \\
& +\frac{t^{2}-2 t-3}{32}\left(|\theta|^{2} \psi_{0}, \psi_{0}\right)-\frac{t+3}{4} \Re\left(\theta \square \psi_{0}, \psi_{0}\right) .
\end{aligned}
$$

Proof: It follows by (2.18) that

$$
\left|P_{0}^{t} \psi_{0}\right|^{2}=\left|\nabla^{t} \psi_{0}\right|^{2}-\left|D^{t} \psi_{0}\right|^{2}-\frac{1}{4} \sum_{j=1}^{4}<e_{j} \bar{p}\left(e_{j}\right) D^{t} \psi_{0}, D^{t} \psi_{0}>
$$


But $\sum_{j=1}^{4} e_{j} \bar{p}\left(e_{j}\right)=\frac{1}{2}\left(-4+i \sum_{j=1}^{4} e_{j} J e_{j}\right)=-2-i \Omega$. Thus $\sum_{j=1}^{4} e_{j} \bar{p}\left(e_{j}\right) D^{t} \psi_{0}=-2 D^{t} \psi_{0}$ since $D^{t} \psi_{0} \in \Gamma\left(\Sigma_{1} M\right)$. Hence,

$$
\left|P_{0}^{t} \psi_{0}\right|^{2}=\left|\nabla^{t} \psi_{0}\right|^{2}-\frac{1}{2}\left|D^{t} \psi_{0}\right|^{2}
$$

By (2.15) and (2.16) we obtain

$$
D^{t} \psi_{0}=\square \psi_{0}+\frac{t-1}{4} \theta \psi_{0}
$$

and hence

$$
\left|D^{t} \psi_{0}\right|^{2}=\left|\square \psi_{0}\right|^{2}+\frac{(t-1)^{2}}{16}<|\theta|^{2} \psi_{0}, \psi_{0}>-\frac{t-1}{2} \Re<\theta \square \psi_{0}, \psi_{0}>
$$

By (2.13) we obtain

$$
\nabla_{X}^{t} \psi_{0}=\nabla_{X}^{-1} \psi_{0}+\frac{t+1}{4} i \theta(J X) \psi_{0}
$$

Hence,

$$
\left|\nabla^{t} \psi_{0}\right|^{2}=\left|\nabla^{-1} \psi_{0}\right|^{2}+\frac{(t+1)^{2}}{16}<|\theta|^{2} \psi_{0}, \psi_{0}>+\frac{t+1}{2} \Re<i \nabla_{J \theta^{\#}}^{-1} \psi_{0}, \psi_{0}>
$$

It is easily seen that $D^{t} \circ \alpha+\alpha \circ D^{t}=\sum_{j=1}^{4} e^{j} \nabla_{e_{j}}^{t} \alpha-2 \nabla_{\alpha \#}^{t}$ for arbitrary 1-form $\alpha$. Hence

$$
\begin{aligned}
<\nabla_{J \theta \#}^{t} \psi_{0} & , \quad \psi_{0}> \\
& =\frac{1}{2} \sum_{j=1}^{4}<e^{j} \nabla_{e_{j}}^{t}(J \theta) \psi_{0}, \psi_{0}>-\frac{1}{2}<D^{t} \circ J \theta \psi_{0}, \psi_{0}>-\frac{1}{2}<J \theta \circ D^{t} \psi_{0}, \psi_{0}> \\
& =\frac{i}{2} \sum_{j=1}^{4}<e^{j} \nabla_{e_{j}}^{t} \theta \psi_{0}, \psi_{0}>-\frac{i}{2}<D^{t} \circ \theta \psi_{0}, \psi_{0}>+\frac{i}{2}<\theta \circ D^{t} \psi_{0}, \psi_{0}> \\
& =i<\theta \circ D^{t} \psi_{0}, \psi_{0}>+i<\nabla_{\theta \#}^{t} \psi_{0}, \psi_{0}>.
\end{aligned}
$$

Substituting this equation in (3.27) and using (3.24) and the fact that

$$
\Re<\nabla_{\theta^{\#}}^{t} \psi_{0}, \psi_{0}>=\frac{1}{2}\left(<\nabla_{\theta^{\#}}^{t} \psi_{0}, \psi_{0}>+<\psi_{0}, \nabla_{\theta^{\#}}^{t} \psi_{0}>\right)=\frac{1}{2} \theta^{\#}\left(\left|\psi_{0}\right|^{2}\right)=\frac{1}{2} g\left(\theta, d\left(\left|\psi_{0}\right|^{2}\right)\right),
$$

we obtain

$$
\begin{aligned}
\left|\nabla^{t} \psi_{0}\right|^{2}= & \left|\nabla^{-1} \psi_{0}\right|^{2}+\frac{t^{2}-2 t-3}{16}<|\theta|^{2} \psi_{0}, \psi_{0}>-\frac{t+1}{4} g\left(\theta, d\left(\left|\psi_{0}\right|^{2}\right)\right) \\
& -\frac{t+1}{2} \Re<\theta \square \psi_{0}, \psi_{0}>.
\end{aligned}
$$

Now (3.22) is obtained by substituting (3.25) and (3.28) in (3.23), integrating and using (2.20) to express $\left\|\nabla^{-1} \psi_{0}\right\|^{2}=\left(\Delta^{-1} \psi_{0}, \psi_{0}\right)=\left(\square^{2} \psi_{0}, \psi_{0}\right)-\frac{1}{4}\left(\left(s-\delta \theta-\frac{|\theta|^{2}}{2}\right) \psi_{0}, \psi_{0}\right)$.

Lemma 3.3 Let $P_{0}^{t} \psi_{0}=0$, where $\psi_{0} \in \Gamma\left(\Sigma_{0} M\right)$ is non-identically zero and $t \neq 1$. Then $(M, g, J)$ is locally conformally Kähler. 
Proof: By (2.19) we have $\nabla_{Z}^{t} \psi_{0}=0$ for $Z \in T^{1,0} M$. Hence, the curvature $R^{t}\left(Z_{1}, Z_{2}\right) \psi_{0}=0$ for $Z_{1}, Z_{2} \in T^{1,0} M$. It follows by Lemma 3.2 that the principal symbol of $\left(P_{0}^{t}\right)^{*} P_{0}^{t}$ is multiple of identity and thus by theorem of Aronszjan [2] $\psi_{0} \neq 0$ on dense open subset of $M$. Since $\Sigma_{0} M$ is line bundle, it follows that the curvature form $R^{t}$ is $(1,1)$-form on this dense open subset and hence on the whole $M$. By 2.13$)$ we obtain $R^{t}=R^{1}-\frac{t-1}{4} i d(J \theta)$. But $R^{1}$ is the curvature of the Chern connection and hence is also a $(1,1)$-form. Thus $d J \theta$ is $(1,1)$-form and therefore $d \theta$ is $(1,1)$-form. Since $d \theta \perp \Omega$ it follows that $d \theta$ is anti-self-dual and the compactness of $M$ implies that $d \theta=0$, i.e. $(M, g, J)$ is locally conformally Kähler.

Proof of Theorem 1.3: By theorem of Gauduchon [9] there always exists a metric $\widetilde{g}$ in the conformal class of $g$ whose Lee form is co-closed. By (2.4) $\widetilde{k}>0$ and by (2.3) and (2.5) it follows that $\widetilde{s}>0$. Hence, Theorem 1.1 tels us that $\operatorname{Ker}(\square)=\{0\}$, i.e. $\lambda \neq 0$. Thus, by Lemma 3.1 there exists an eigenspinor $\psi_{0} \in \Gamma\left(\Sigma_{0} M\right)$ of $\square^{2}$ with respect to $\lambda^{2}$. Applying Lemma 3.2 with $t=-3$ we obtain

$$
\left(\square^{2} \psi_{0}, \psi_{0}\right)=2\left\|P_{0}^{-3} \psi_{0}\right\|^{2}+\frac{1}{2}\left(\left(s-3 \delta \theta-\frac{3}{2}|\theta|^{2}\right) \psi_{0}, \psi_{0}\right) .
$$

But it follows by (2.3) and (2.5) that $s-3 \delta \theta-\frac{3}{2}|\theta|^{2}=k$. Thus

$$
\lambda^{2}\left\|\psi_{0}\right\|^{2}=2\left\|P_{0}^{-3} \psi_{0}\right\|^{2}+\frac{1}{2}\left(k \psi_{0}, \psi_{0}\right)
$$

Hence, $\lambda^{2} \geq \frac{1}{2} \inf _{M} k$ and the equality is attained iff $P_{0}^{-3} \psi_{0}=0$ and $k=$ const.

The last statement of the theorem follows from Lemma 3.3.

Remark: In Lemma 3.3 we characterize the compact Hermitian spin surfaces admitting Hermitian twistor spinor with respect to any of the canonical Hermitian connections except the Chern connection. In the same direction, one can see that under the additional assumptions of $b_{1}$ odd and positive fundamental constant $C(M, g)$ (see the definition in [10) a compact Hermitian spin surface admitting Hermitian twistor spinor with respect to the Chern connection is biholomorphic to a primary Hopf surface. To prove this notice that the positivity of $C(M, g)$ implies by Gauduchon's plurigenera theorem [10] that all the plurigenera of $(M, J)$ vanish and since $b_{1}$ is odd $(M, J)$ must be of type $V I I_{0}$. A Hermitian twistor spinor with respect to the Chern connection is an antiholomorphic section $\psi$ of $K^{\frac{1}{2}}$. Equivalently, $j \psi$ is holomorphic section of $K^{-\frac{1}{2}}$ and hence $H^{0}\left(M, \mathcal{O}\left(K^{-1}\right)\right) \neq 0$. Now the statement follows by the fact that $M$ is spin and arguments similar to those in Corollary 3.12 in [19].

\section{Examples}

In this section we give non-Kähler examples of Hermitian surfaces for which the limiting case in Theorem 1.3 is attained.

Recall (cf. 四) that under a conformal change of the metric $\widetilde{g}=e^{f} g$ of a spin manifold $(M, g)$ there is an identification $\sim$ of the spinor bundle $\Sigma M$ of $(M, g)$ and the spinor bundle $\widetilde{\Sigma} M$ of $(M, \widetilde{g})$ such that

$$
\begin{gathered}
\widetilde{X \psi}=\widetilde{X} \widetilde{\psi} \\
\widetilde{\alpha \psi}=\widetilde{\alpha} \widetilde{\psi} \\
\widetilde{\nabla}_{X} \widetilde{\psi}=\widetilde{\nabla_{X} \psi}-\frac{1}{4} X d f \widetilde{\psi}-\frac{1}{4} X f . \widetilde{\psi}
\end{gathered}
$$


where $\psi \in \Gamma(\Sigma M), \widetilde{\psi} \in \Gamma(\widetilde{\Sigma} M)$ is the spinor corresponding to $\psi, X \in T M, \widetilde{X}=e^{-\frac{f}{2}} X$, $\alpha \in T^{*} M, \widetilde{\alpha}=e^{\frac{f}{2}} \alpha$, and $\nabla$ and $\widetilde{\nabla}$ are the Levi-Civita connections of $g$ and $\widetilde{g}$ respectively.

Now, if $(M, g, J)$ is a Hermitian surface, the Kähler form of $(M, \widetilde{g}, J)$ is $\widetilde{\Omega}=e^{f} \Omega$ and it follows from (4.30) that

$$
\widetilde{\Omega \psi}=\widetilde{\Omega} \widetilde{\psi}
$$

Thus the eigensubbundles $\Sigma_{r} M$ of $\Omega$ correspond to the eigensubbundles $\widetilde{\Sigma}_{r} M$ of $\widetilde{\Omega}$. The Lee form of $\widetilde{g}$ is $\theta+d f$ and hence by (2.13), (4.29)-(4.32) we obtain

$$
\widetilde{\nabla}_{X}^{t} \widetilde{\psi_{0}}=\widetilde{\nabla_{X}^{t} \psi_{0}}-\frac{t+1}{4} i(J d f)(X) \widetilde{\psi_{0}}, \quad \psi_{0} \in \Gamma\left(\Sigma_{0} M\right)
$$

Lemma 4.1 Let $(M, g, J)$ be a Hermitian spin surface and $\widetilde{g}=e^{f} g$. If $\psi_{0} \in \Gamma\left(\Sigma_{0} M\right)$ is a Hermitian twistor spinor with respect to $\nabla^{t}$ then $e^{\frac{t+1}{4} f} \widetilde{\psi_{0}}$ is a Hermitian twistor spinor with respect to $\widetilde{\nabla}^{t}$.

Proof: Easy consequence of (2.19) and (4.33).

Now let $M$ be one of the manifolds $S^{2} \times S^{2}$ and $T^{2} \times S^{2}$, where $S^{2}$ is the 2-dimensional sphere and $T^{2}$ is 2-dimensional flat torus. With their standard product metrics these manifolds are limiting Kähler manifolds, i.e. they are Kähler and (1.2) is equality for them (cf. [16] or [8]). The limiting Kähler manifolds are characterized by constant positive scalar curvature and existence of Kählerian twistor spinor, i.e. an antiholomorphic section of $K^{\frac{1}{2}}$ - the square root of the canonical line bundle which determines the spin structure (cf. [17] or Theorem 1.3 above).

We can change conformally the metric of the first factor of $M$ so to obtain a metric $g$ on $M$ of positive non-constant scalar curvature $s$. This metric will be Kähler with respect to the same complex structure $J$ and $M$ will still admit Kählerian twistor spinor. Now we change the metric conformally by $\widetilde{g}=s g$. Since in the Kähler case the connections $\nabla^{t}$ coincide with the Levi-Civita connection, it follows from Lemma 4.1 that on $(M, \widetilde{g}, J)$ there exists a Hermitian twistor spinor with respect to $\nabla^{t}$ (and in particular $\nabla^{-3}$ ) and by (2.4) the conformal scalar curvature is constant: $\widetilde{k}=1$. Thus by Theorem 1.3 the inequality (1.2) turns into equality for the non-Kähler Hermitian surface $(M, \widetilde{g}, J)$.

\section{References}

[1] V.Apostolov, J.Davidov, O.Muškarov, Self-dual Hermitian surfaces, Trans. Amer. Math. Soc. 349 (1996), $3051-3063$.

[2] N.Aronszjan, A unique continuation theorem for solutions of elliptic partial differential equations or inequalities of second order, J. Math. Pures Appl. 35 (1957), 235 - 249.

[3] M.F.Atiyah, R.Bott, A.Shapiro, Clifford modules, Topology 3 (Suppl. 1) (1964), 3 - 38.

[4] H.Baum, T.Friedrich, R.Grunewald and I.Kath, Twistor and Killing spinors on Riemannian manifolds, Seminarbericht 108, Humboldt Universität Berlin, 1990.

[5] J.-M.Bismut, A local index theorem for non-Kähler manifolds, Math. Ann. 284 (1989), $681-699$. 
[6] C.Boyer, A note on hyperhermitian four manifolds, Proc. Amer. Math. Soc. 102 No 1 (1988), $157-164$.

[7] T.Friedrich, Der erste Eigenwert des Dirac operators einer kompakten Riemannischen Manningfaltigkeit nichtnegativer Skalarkrümung, Math. Nachrichten 97 (1980), 117 - 146.

[8] T.Friedrich, The classification of 4-dimensional Kähler manifolds with small eigenvalue of the Dirac operator, Math. Ann. 295 (1993), 565 - 574.

[9] P.Gauduchon, Le theoreme de l'excentricité nulle, C. R. Acad. Sci. Paris Ser. A 285 (1977), $387-390$.

[10] P.Gauduchon, Fibres Hermitiens à endomorphisme de Ricci non négatif, Bul. Soc. Math. France 105 (1977), 113 - 140.

[11] P.Gauduchon, La 1-forme de torsion d'une variété hermitienne compacte, Math. Ann. 267 (1984), $495-518$.

[12] P.Gauduchon, Hermitian connections and Dirac operators, Bol. U. M. I. ser. VII, vol. XI-B, supl. 2 (1997), $257-289$.

[13] O.Hijazi, Opérateurs de Dirac sur le variétés riemanniennes: Minoration des valeurs propres, Thèse de 3ème Cycle, Ecole Polytechnique (1984).

[14] N.Hitchin, Harmonic spinors, Adv. Math. 14 (1974), 1 - 55.

[15] K.-D.Kirchberg, An estimation for the first eigenvalue of the Dirac operator on closed Kähler manifolds of positive scalar curvature, Ann. Glob. Anal. Geom. 4 (1986), 291 - 325.

[16] K.-D.Kirchberg, The first eigenvalue of the Dirac operator on Kähler manifolds, J. Geom. Phys. 7 (1990), $447-468$.

[17] K.-D.Kirchberg, Properties of Kählerian twistor-spinors and vanishing theorems, Math. Ann. 293 (1992), $349-369$.

[18] A.Moroianu, Structures de Weyl admettant des spineurs parallèles, Bull. Soc. Math. Fr. 124, N4, (1996), $685-695$.

[19] M.Pontecorvo, Complex structures on Riemannian four-manifolds, Math. Ann. 309, (1997), $159-177$.

[20] I.Vaisman, Some curvature properties of complex surfaces, Ann. Mat. Pura Appl. 132, (1982), $1-18$.

[21] M.Wang, Parallel spinors and parallel forms, Ann. Glob. Anal. Geom. 7 (1989), 59 - 68.

\section{Authors' permanent address}

Bogdan Alexandrov, Gueo Grantcharov, Stefan Ivanov

University of Sofia, Faculty of Mathematics and Informatics, Department of Geometry,

5 James Bourchier blvd, 1126 Sofia, BULGARIA.

E-mail: B.Alexandrov: alexandrovbt@fmi.uni-sofia.bg 
G.Grantcharov: geogran@fmi.uni-sofia.bg

S.Ivanov: ivanovsp@fmi.uni-sofia.bg

Present address of Gueo Grantcharov:

Department of Mathematics, University of Californiya at Riverside, Riverside, CA 92521. 